\title{
An X-ray Study of the Heusler-type Ordering in $\mathrm{AuAgZn}_{2}$ Alloy
}

\author{
By Yuichiro Murakami*, Yasuhiro Watanabe** and Sukeji Kachi*
}

\begin{abstract}
The kinetic behavior of the Heusler( $\left(2_{1}\right)$-type ordering in the stoichiometric $\mathrm{AuAgZn_{2 }}$ alloy quenched from the $\mathrm{CsCl}(\mathrm{B} 2)$-type ordered region was studied by X-ray diffraction. The degrees of the B2-type order $\left(S_{1}\right)$ and the $\mathrm{L} 2{ }_{1}$-type order $\left(S_{H}\right)$ and the domain size of the L2 ${ }_{1}$-type superstructure were measured as a function of annealing time during the isothermal ordering process at 366, 386 and $406 \mathrm{~K}$. The isothermal change of $S_{H}$ was analyzed by the standard rate equation and the activation energy of the L $2_{1}$-type ordering was found to be $46 \mathrm{~kJ} / \mathrm{mol}$. The increase of the L2 $2_{1}$-type domain size was proportional to the three-tenth power of the annealing time. The temperature dependence of $S_{1}$ and $S_{H}$ were also studied. $S_{1}$ showed a minimum whereas $S_{H}$ decreased to zero at the critical temperature of the $\mathrm{L} 2{ }_{1}$-type order on heating.
\end{abstract}

(Received May 17, 1980)

\section{Introduction}

Noble metal based- $\beta$ phase alloys such as $\mathrm{AuAgZn} \mathrm{n}_{2}$ and $\mathrm{AuCuZn}_{2}$ have been reported to have two types of superstructure, i.e. the CsCl(B2)-type at high temperatures and the Heusler(L2 1 )-type at low temperatures ${ }^{(1) \sim(4)}$; The phase diagram of $\mathrm{Au}_{x} \mathrm{Ag}_{50-x} \mathrm{Zn}_{50}$ alloy is shown in Fig. 1 as an example. The L2 ${ }_{1}$-type ordering has been treated by the statistical thermodynamics and it was shown that the difference of the critical temperatures $\left(T_{\mathrm{c} 2}\right)$ of the $\mathrm{L2}_{1}$ lattice between $\mathrm{AuAgZn} \mathrm{Z}_{2}$ and $\mathrm{AuCuZn} \mathrm{Z}_{2}$ alloys is due to the difference of the ordering energies between $\mathrm{Au}-\mathrm{Ag}$ and $\mathrm{Au}-\mathrm{Cu}$ pairs of the next nearest neighbors ${ }^{(4)}$. Muldawer has shown that the $\mathrm{L} 22_{1}$-type ordering cannot be suppressed even by quenching from high temperature in $\mathrm{AuCuZn}_{2}$ alloy, whereas it can be suppressed partially in $\mathrm{AuAgZn}_{2}$ alloy $^{(1)}$. Then the stoichiometric $\mathrm{AuAgZn}_{2}$ alloy seems to be appropriate for a study of the kinetic behavior of the L2 2 -type ordering.

The kinetics of the ordering in the $b c c$ alloy has been studied for the $\mathrm{DO}_{3}$-type $\mathrm{Fe}_{3} \mathrm{Al}$ alloy by many investigators ${ }^{(5) \sim(8)}$. The degree of the $\mathrm{DO}_{3}$-type order and the size of the

* Department of Chemistry, Faculty of Science, Kyoto University, Kyoto 606, Japan.

** Graduate School, Kyoto University, Kyoto. Present address: Department of Chemistry, Saitama University, Urawa 338, Japan. ordered domains were measured by X-ray diffraction during the ordering process. For example, Davies ${ }^{(6)}$ reported that the isothermal $\mathrm{DO}_{3}$-type ordering proceeded by the nucleation and growth process of the ordered domains, whereas on slow cooling the $\mathrm{DO}_{3}$-type ordering proceeded by a homogeneous nucleation process. Oki et al. ${ }^{(8)}$ showed that the isothermal $\mathrm{DO}_{3}$-type ordering is caused by the nucleation and growth of the $\mathrm{DO}_{3}$-type domain in the $\mathrm{B} 2$ matrix and that the activation energy is 155 $\mathrm{kJ} / \mathrm{mol}$.

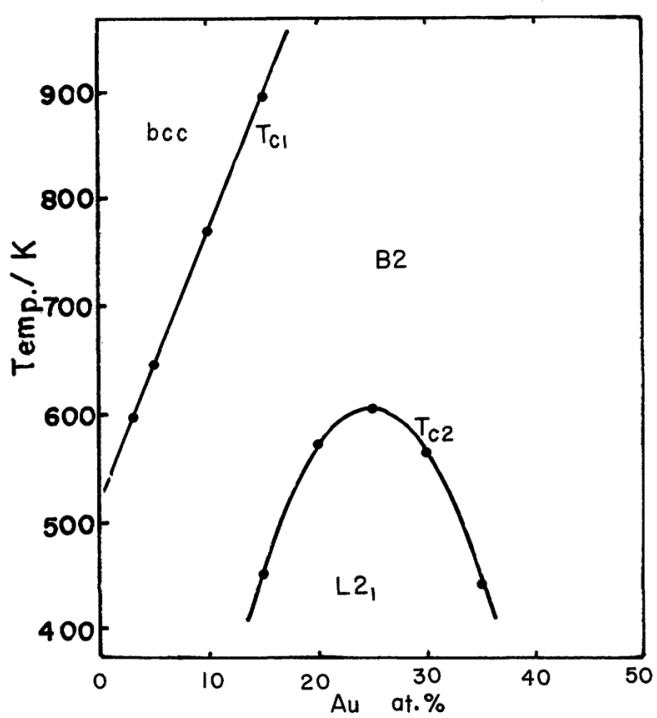

Fig. 1 Critical temperature of the B2-type structure $\left(T_{\mathrm{c} 1}\right)$ and that of the $\mathrm{L} 22_{1}$-type structure $\left(T_{\mathrm{c} 2}\right)$ in $\mathrm{Au}_{x} \mathrm{Ag}_{50-x} \mathrm{Zn}_{50}$ alloys (after Brooks et al. ${ }^{(2)(4)}$ ). 
In the $\mathrm{L} 2{ }_{1}$-type $\mathrm{AuCuZn}{ }_{2}$ alloy, the degree of the B2-type order $S_{1}$ was reported to decrease to zero during the disordering process $^{(3)}$. In the stoichiometric L2 ${ }_{1}$-type $\mathrm{AuAgZn}{ }_{2}$ alloy, the temperature dependence of the degree of the $\mathrm{L} 2{ }_{1}$-type order $S_{H}$ was reported by Muldawer ${ }^{(1)}$ but the information about the kinetic behavior has not been available. Therefore, in this study, the kinetic behaviors of the stoichiometric $\mathrm{AuAgZn}{ }_{2}$ alloy quenched from the $\mathrm{B} 2$ region are investigated by $\mathrm{X}$-ray diffraction during the isothermal ordering process from the B2- to L2 1 -type superstructures, and the isothermal change of $S_{1}$ and $S_{H}$ is analyzed quantitatively using a standard rate equation given by Feder et al. ${ }^{(7) \sim(9)}$

\section{Experimental}

The alloy specimens were prepared by melting metals with high purity $(99.99 \% \mathrm{Au}$, $\mathrm{Ag}$ and $99.999 \% \mathrm{Zn}$ ) in an evacuated quartz tube. The specimens were annealed at $873 \mathrm{~K}$ for $288 \mathrm{ks}$ and then quenched into salt brine $(267 \mathrm{~K})$. The alloy ingot was filed into powders finer than $74 \mu \mathrm{m}$. The powdered specimens, sealed in quartz tubes, were annealed at $723 \mathrm{~K}$ (in the $\mathrm{B} 2$ region) for $3.6 \mathrm{ks}$ and then quenched to $267 \mathrm{~K}$. After this heat treatment, powdered specimens were annealed at temperatures 366,386 and $406 \mathrm{~K}$, well below $T_{\mathrm{c} 2}(=588 \mathrm{~K})$ of the L2 $2_{1}$-type ordering, for a definite period and then quenched to $267 \mathrm{~K}$. Variation of the degree of the B2- and L2 ${ }_{1}$-type order, $S_{1}$ and $S_{H}$, were measured by X-ray diffraction using a filtered $\mathrm{CuK}_{\alpha}$ radiation at room temperature. The temperature dependences of $S_{1}$ and $S_{H}$ were also studied using a high temperature $\mathrm{X}$-ray apparatus.

\section{Analysis of the Degree of Order}

The unit cell of the L2 -type superlattice, which consists of three kinds of sublattices, $\alpha, \beta$ and $\gamma$, is shown in Fig. 2 . In the completely ordered state, $\alpha, \beta$ and $\gamma$ sites are occupied, respectively, by $\mathrm{Zn}, \mathrm{Au}$ and $\mathrm{Ag}$ atoms. In a previous paper ${ }^{(4)}$, in order to describe the occupation probabilities of the constituent atoms in the three sublattice sites, four kinds

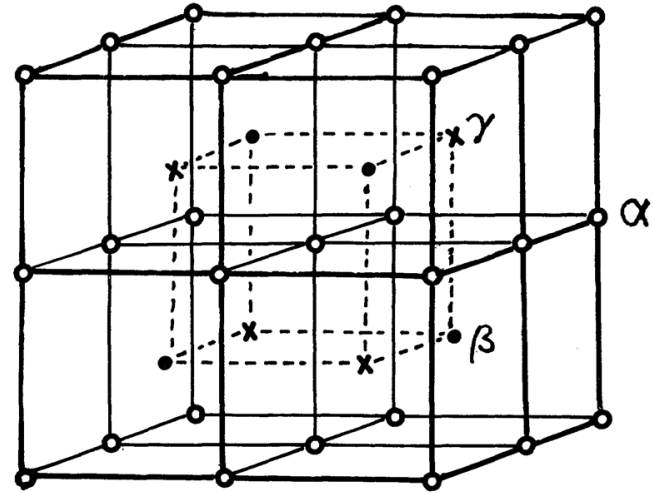

Fig. 2 Unit cell of the Heusler-type( $\left(2_{1}\right)$ superlattice which consists of three kinds of sublattices, $\alpha, \beta$ and $\gamma$.

of the degree of order were defined as follows:

$$
\begin{aligned}
& S_{1}=2\left(A_{\alpha}-x_{\mathrm{A}}\right), S_{2}=4\left(B_{\beta}-x_{\mathrm{B}}\right) / 3, \\
& S_{3}=4\left(C_{\gamma}-x_{\mathrm{C}}\right) / 3 \text { and } S_{4}=2\left(x_{\mathrm{A}}-A_{\beta}\right),
\end{aligned}
$$

where $x_{\mathrm{A}}, x_{\mathrm{B}}$ and $x_{\mathrm{C}}$ represent the mole fraction of $\mathrm{Zn}, \mathrm{Au}$ and $\mathrm{Ag}$ atoms and $A_{\alpha}, B_{\beta}$ and $C_{\gamma}$ the probabilities of finding a $\mathrm{Zn}$ atom on the $\alpha$ site, $\mathrm{Au}$ atom on the $\beta$ site and $\mathrm{Ag}$ atom on the $\gamma$ site, respectively.

The degree of order can be obtained by measuring integrated intensity of the X-ray reflections. The intensity $I_{h k l}$, from $(h k l)$ plane is given by the following formula:

$$
I_{h k l}=c m L(\theta) F_{h k l}^{2} \exp \left(-\gamma \sin ^{2} \theta\right),
$$

where $c$ is a constant, $m$ the multiplicity factor, $L(\theta)$ the Lorentz-polarization factor, $F_{h k l}$ the structure factor and $\theta$ the Bragg angle. $\gamma$, the temperature factor, is determined by plotting $\ln \left\{I_{h k l} / m L(\theta) F_{h k l}\right\}$ of the fundamental reflections against $\sin ^{2} \theta$ as suggested by Oki et al. ${ }^{(8)}$

The structure factor $F_{h k l}$ for the ternary $b c c$ alloy is given by the following equations:

(i) When $h, k, l$ are all even and $(h+k+1) / 2$ is even, i.e. for the fundamental reflections,

$$
F_{h k l}=4 x_{\mathrm{A}} f_{\mathrm{Zn}}+4 x_{\mathrm{B}} f_{\mathrm{Au}}+4 x_{\mathrm{C}} f_{\mathrm{Ag}},
$$

where $f_{i}$ represents the atomic scattering factor of the $i$-th atom.

(ii) When $h, k, l$ are all even and $(h+k+l) / 2$ is odd, i.e. for the B2-type superlattice reflections, 


$$
F_{h k l}=\left\{2 f_{\mathrm{Zn}}-\left(x_{\mathrm{B}} f_{\mathrm{Au}}+x_{\mathrm{C}} f_{\mathrm{Ag}}\right) /\left(1-x_{\mathrm{A}}\right)\right\} S_{1},
$$

where $S_{1}$ represents the degree of the B2-type order.

(iii) When $h, k, l$ are all odd, i.e. for the L2 ${ }_{1}$-type superlattice reflections,

$$
\begin{gathered}
F_{h k l}=\left(f_{\mathrm{Au}}-f_{\mathrm{Ag}}\right) S_{H}, \\
\text { and } S_{H}=3\left(S_{2}+S_{3}\right) / 4-S_{1} / 2,
\end{gathered}
$$

where $S_{H}$ represents the degree of the $\mathrm{L} 2{ }_{1}$-type order.

Each value of the four parameters, $S_{1}, S_{2}$, $S_{3}$ and $S_{4}$, in eq. (1) cannot be determined directly by the X-ray measurement. Thereby, in the previous paper ${ }^{(4)}$, the relations between the probabilities such as $A_{\beta}=A_{\gamma}$ and $B_{\alpha} / x_{\mathrm{B}}=$ $C_{\alpha} / x_{\mathrm{C}}$ were assumed, which gave simple relations between order parameters in the stoichiometric $\mathrm{A}_{2} B C$ composition as follows:

$$
S_{1}=S_{4} \text { and } S_{2}=S_{3} .
$$

In this paper, $S_{1}$ and $S_{H}$ are used as the parameters, because they can be determined directly by an X-ray analysis. In the B2-type lattice, $S_{1}=1$ and $S_{2}=S_{3}=1 / 3^{(4)}$, yielding $S_{H}=0$. In the L2 ${ }_{1}$ lattice, $S_{1}=1$ and $S_{2}=S_{3}=1$, yielding $S_{H}=1$. Then the new parameter $S_{H}$ defined by eq. (6) seems to be appropriate to describe the degree of the $\mathrm{L} 2{ }_{1}$-type order. The parameters, $S_{2}, S_{3}$ and $S_{4}$, can be calculated from $S_{1}$ and $S_{H}$ using eqs. (6) and $\left(1^{\prime}\right)$ in the case of the $\mathrm{Au}-\mathrm{Ag}-\mathrm{Zn}$ system $^{(4)}$.

$S_{1}$ and $S_{H}$ of $\mathrm{AuAgZn} \mathrm{Al}_{2}$ alloy were determined from the ratio of the integrated intensities of the superlattice and the fundamental reflections, $I_{111} / I_{220}$ and $I_{200} / I_{220}$, through eqs. (2) (5) using the measured quantities at room temperature. The temperature dependences of $S_{1}$ and $S_{H}$ were determined, respectively, by comparing the experimental values of $\left(I_{200} / I_{220}\right)$ and $\left(I_{111} / I_{220}\right)$ at high temperatures with the calculated values of those for the completely ordered state ${ }^{(1)}$, i.e. by $\left(I_{111} / I_{220}\right)_{\mathrm{exp}} /\left(I_{111} / I_{220}\right)_{\mathrm{calc}}=S_{H}^{2}$ and $\left(I_{200} /\right.$ $\left.I_{220}\right)_{\text {exp }} /\left(I_{200} / I_{220}\right)_{\text {calc }}=S_{1}^{2}$.

The size of the $\mathrm{L} 2{ }_{1}$-type ordered domain, $D$, is obtained by using Scherrer's equation:

$$
D=\lambda / \beta \cos \theta \text { and } \beta=\left(B^{2}-b^{2}\right)^{0.5},
$$

where $\lambda$ is the wavelength of X-ray, $\theta$ the Bragg angle, $B$ the half width of the (111) reflection, and $b$ the half width due to the instrumetnal broadening; $b$ is estimated from the half width of the superlattice reflections of the well annealed specimen.

\section{Results and Discussion}

\section{Variation during isothermal annealing}

The degree of order, $S_{1}$ and $S_{H}$, of the stoichiometric $\mathrm{AuAgZn} \mathrm{A}_{2}$ alloy quenched from the $\mathrm{B} 2$ phase $(723 \mathrm{~K})$ were measured during the isothermal $\mathrm{B} 2$-to- $\mathrm{L} 2{ }_{1}$ ordering process by an X-ray diffraction. Isothermal changes of $S_{1}$ and $S_{H}$ at 366,386 and $406 \mathrm{~K}$ are shown in

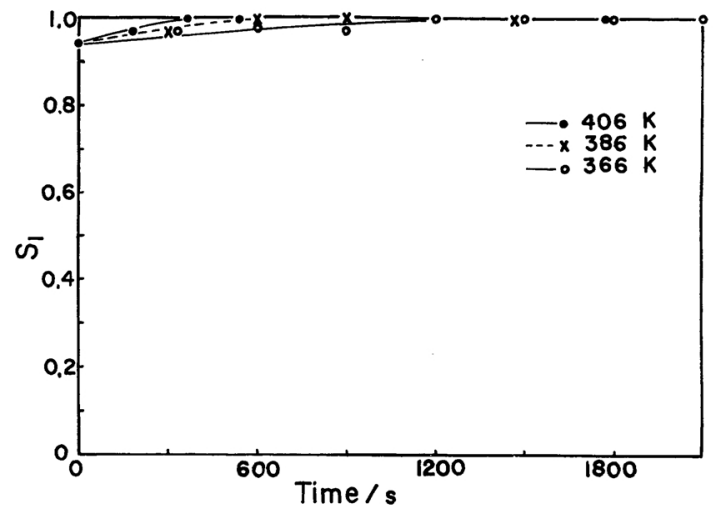

Fig. 3 Isothermal change of the degree of the B2type order $S_{1}$ at temperatures, 366, 386 and $406 \mathrm{~K}$, in the $\mathrm{AuAgZn}$ alloy quenched from $723 \mathrm{~K}$ measured at room temperature.

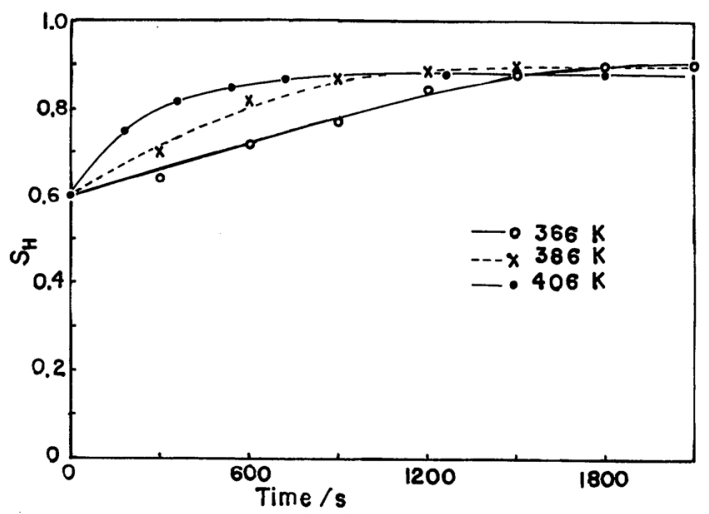

Fig. 4 Isothermal change of the degree of the $\mathrm{L} 2_{1}$ type order $S_{H}$ at temperatures, 366386 and $406 \mathrm{~K}$, in the $\mathrm{AuAgZn} \mathrm{A}_{2}$ alloy quenched from $723 \mathrm{~K}$ measured at room temperature. 
Figs. 3 and 4 . In the quenched specimen, $S_{1}$ is 0.94 , that is near to the equilibrium value $(1.0$ at $293 \mathrm{~K}) . S_{H}$ is only 0.60 ; this result indicates that the $\mathrm{L} 2{ }_{1}$-type ordering is partially suppressed by quenching as pointed out by Muldawer ${ }^{(1)}$. During the isothermal annealing, $S_{1}$ and $S_{H}$ increase rapidly with annealing time and become almost constant after annealing over $2.4 \mathrm{ks}$. Isothermal change of $S_{H}$ is analyzed by the standard rate equation given by Feder et al. ${ }^{(7)(8)}$ :

$$
d S_{H} / d t=\left(S_{H}^{e}-S_{H}\right)^{n} / \tau_{n}
$$

where $S_{H}^{e}$ is the equilibrium value of $S_{H}$ and $\tau_{n}$ the relaxation time for the $n$-th order reaction. From eq. (8), change of $S_{H}$ is shown as a function of annealing time $t$ as follows:

$$
\begin{gathered}
\left(\frac{S_{H}^{e}-S_{H}}{S_{H}^{e}-S_{H}^{0}}\right)^{1-n}=1+t(n-1)\left(S_{H}^{e}-S_{H}^{0}\right)^{n-1} / \tau_{n} \\
\text { and } \tau_{n}=\tau_{1}(n-1)\left(S_{H}^{e}-S_{H}^{0}\right)^{n-1} /\{\exp (n-1)-1\} \text {, }
\end{gathered}
$$

where $S_{H}^{0}$ is the value of $S_{H}$ of the quenched specimen (i.e. at $t=0$ ) and $\tau_{1}$ the relaxation time of the 1 st order reaction ${ }^{(7)}$. The isothermal change of $S_{H}$ shown in Fig. 4 can be well explained by this standard rate equation using parameters shown in Table 1.

Activation energy for the $\mathrm{L} 2{ }_{1}$-type ordering is obtained from the Arrhenius plot, i.e. a plot of $\ln \tau_{n}$ as a function of $1 / T$ as shown in Fig. 5 . From the slope of a straight line in Fig. 5, the activation energy $Q$ of the $\mathrm{L} 2{ }_{1}$-type ordering was found to be $46 \pm 2 \mathrm{~kJ} / \mathrm{mol}$, which is considerably smaller than that $(155 \mathrm{~kJ} / \mathrm{mol})$ of the $\mathrm{DO}_{3}$-type ordering in $\mathrm{Fe}_{3} \mathrm{Al}$ alloy ${ }^{(8)}$. In the binary $\beta$ phase alloy such as $\mathrm{AgZn}$ and $\mathrm{AuZn}$, the activation energy for the self diffusion has been reported to be $70 \sim 138$ $\mathrm{kJ} / \mathrm{mol}^{(10)}$. The activation energy of the

Table 1 Relaxation time $\left(\tau_{n}\right)$ and parameters used to fit a standard rate equation to the isothermal ordering process. $\tau_{1}$ is obtained directly from the curves of Fig. 4 assuming $n=1$ in eq. (8).

\begin{tabular}{cccc}
\hline$T / \mathrm{K}$ & $\tau_{1} / \mathrm{s}$ & $\tau_{n} / \mathrm{s}$ & $n$ \\
\hline 366 & $960 \pm 24$ & 932 & 1.02 \\
386 & $480 \pm 12$ & 440 & 1.06 \\
406 & $240 \pm 12$ & 208 & 1.10 \\
\hline
\end{tabular}

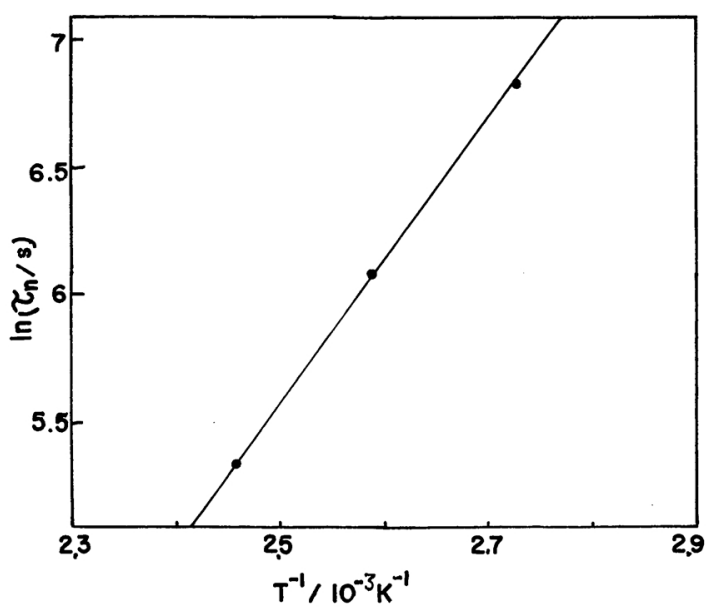

Fig. 5 Relaxation time for the $L 2_{1}$-type ordering as a function of reciprocal absolute temperature.

L2 1 -type ordering obtained here may be smaller than that of the self diffusion in $\mathrm{AuAgZn} \mathrm{n}_{2}$ alloy.

The growth of the L2 1 -type ordered domains during the isothermal annealing is shown in Fig. 6. The change of the half width of the B2-type superlattice reflection could not be observed. The growth of the domain size $D$ in many alloys has been reported to be described by the following equation ${ }^{(8)(13)}$ :

$$
D=k t^{n} \text {, }
$$

where $k$ and $n$ are constants. Generally, $n$ is in a range from 0.1 to $0.5^{(13)}$. From the slope of the straight lines in $\log D$-vs-log $t$ curves in Fig. 6, $n$ is found to be 0.3 . This is in agreement with that $(n=0.3)$ for the growth of the $\mathrm{DO}_{3}$ type domains in $\mathrm{Fe}_{3} \mathrm{Al}^{(8)}$. The size of the $\mathrm{L}_{2}$ type domains increases with annealing time

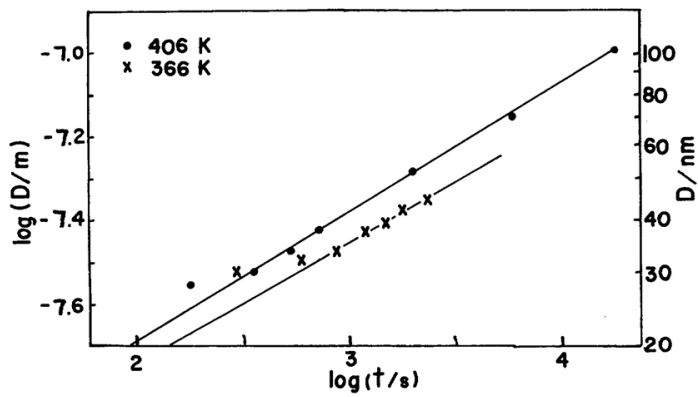

Fig. 6 Growth of the antiphase domain size $D$ as a function of the logarithm of annealing time during isothermal $\mathrm{L} 2{ }_{1}$-type ordering. 
even after the saturation of $S_{H}$ (i.e. after the time longer than $\log (t / s)=3$ ) as shown in Fig. 6. This is similar to the growth of the $\mathrm{DO}_{3}$ domains in the $\mathrm{B} 2$ matrix in $\mathrm{Fe}_{3} \mathrm{Al}$ alloy ${ }^{(8)}$. These results suggest that the $\mathrm{L} 2{ }_{1}$-type ordered domains are nucleated in the B2 matrix and they grow by the movement of domain boundaries even after the saturation of degree of order. The existence of antiphase domains in the Heusler alloy $\mathrm{AuCuZn}_{2}$ has been confirmed by an electron microscopy ${ }^{(12)}$. For $\log (t / s)$ less than about 2.5 in Fig. 6, experimental points deviate from the straight lines; this may be due to the fact that the domain size has a finite value of about $22 \mathrm{~nm}$ even in the quenched specimen (i.e. at $t=0$ ).

The quenched-in defects in the binary $b c c$ alloys such as $\mathrm{CuZn}$ and $\mathrm{AuCd}$ were studied by many investigators ${ }^{(14) \sim(18)}$. By a study of the isothermal annealing process, the activation energy for the migration of vacancies was reported to be $39 \sim 60 \mathrm{~kJ} / \mathrm{mol}$ in these alloys. On the other hand, during the reordering process of the quenched-in disorder in the $\beta$-CuZn alloy, the activation energy for the reordering was reported to be $63 \mathrm{~kJ} / \mathrm{mol}$ by Koczac et al. ${ }^{(16)}$, which is close to the summation of the activation energy for the migration of vacancies $(39 \mathrm{~kJ} / \mathrm{mol})$ and the binding energy of the trapped vacancies $(20 \mathrm{~kJ} / \mathrm{mol})$ at the antiphase domain boundaries ${ }^{(16)(18)}$. The observed activation energy $(46 \mathrm{~kJ} / \mathrm{mol})$ for the $\mathrm{L} 2{ }_{1}$-type ordering in $\mathrm{AuAgZn} \mathrm{n}_{2}$ alloy is of the same order as that for the migration of vacancies in the $\beta$ phase alloys ${ }^{(14)}$. Therefore the vacancies are considered to play an important role in the kinetic behavior of the L2 ${ }_{1}$-type ordering also in $\mathrm{AuAgZn} \mathrm{Al}_{2}$ alloy.

\section{Variation on heating}

In the stoichiometric $\mathrm{AuAgZn}$, alloy, the critical temperature $\left(T_{\mathrm{c} 2}\right)$ of the $\mathrm{L} 2_{1}$-type structure is $587 \mathrm{~K}^{(2)}$ and that $\left(T_{\mathrm{c} 1}\right)$ of the B2-type structure is $1130 \mathrm{~K}^{(4)}$. The variation of $S_{1}$ and $S_{H I}$ of the quenched $\mathrm{AuAgZn}_{2}$ alloy was measured by using a high temperature $\mathrm{X}$-ray apparatus on the step-wise heating process: temperature was raised at a definite interval and then kept constant during the measurement (averaged rate of heating was

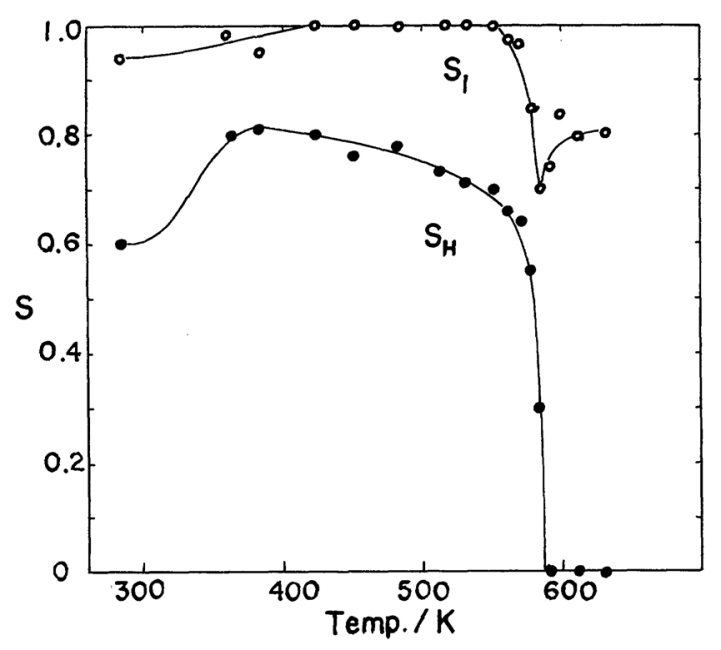

Fig. 7 Variation of the degree of order, $S_{1}$ and $S_{H}$, in $\mathrm{AuAgZn} \mathrm{Al}_{2}$ alloy on a heating process.

$0.1 \mathrm{~K} / \mathrm{s}$ ). The result is shown in Fig. 7. The experimental values of $S_{1}$ and $S_{H}$ first increase in the temperature range near $360 \mathrm{~K}$, where the ordering reaction is expected to proceed on heating. In the temperature region near $T_{\mathrm{c} 2}, S_{H}$ decreases rapidly and becomes zero at $T_{\mathrm{c} 2}$. The temperature dependence of $S_{H}$ just below $T_{\mathrm{c} 2}$ is very steep compared with that expected from the Bragg-Williams theory. On the other hand, $S_{1}$ decreases rapidly at a temperature near $T_{\mathrm{c} 2}$ and it increases again after passing a minimum at $T_{\mathrm{c} 2}$. This result indicates that the diffusion of atoms due to the disordering process of the $\mathrm{L} 2{ }_{1}$-type lattice destroys partially the B2-type order. Therefore, the diffusion of atoms between the 1 st nearest neighbors as well as that between the 2 nd nearest neighbors are expected to have an important role in the disordering process of the $\mathrm{L} 2{ }_{1}$-type lattice, which will be discussed in a following paper ${ }^{(11)}$. Experiment above $600 \mathrm{~K}$ was very difficult because of the evaporation of $\mathrm{Zn}$ atoms.

\section{Summary}

Kinetic behavior of the $\mathrm{AuAgZn}_{2}$ alloy quenched from the $\mathrm{B} 2$ region was studied by $\mathrm{X}$-ray diffraction. The results are summarized as follows:

(1) The variation of $S_{H}$ during the isothermal ordering process is well explained by the 
standard rate equation and the activation energy of the $\mathrm{L} 22_{1}$-type ordering is found to be $46 \pm 2 \mathrm{~kJ} / \mathrm{mol}$.

(2) The isothermal L2 ${ }_{1}$-type ordering proceeds by the nucleation and growth of the domains in the B2 matrix. The increase of the $\mathrm{L} 2$ domain size is proportional to the three tenth power of the annealing time at a temperature near $400 \mathrm{~K}$.

(3) The temperature dependence of $S_{1}$ and $S_{H}$ were also studied by the high temperature X-ray analysis. $S_{H}$ becomes zero at $T_{\mathrm{c} 2}$ upon heating, whereas $S_{1}$ showed a minimum at $T_{\mathrm{c} 2}$. Diffusion of atoms between the 1 st and the 2 nd nearest neighbors are shown to play an important role in the ordering process in the Heusler alloy.

\section{Acknowledgment}

This work was partly supported by the grantin-aid for the fundamental chemical research from the Japanese Foundation for Scientific Promotion.

\section{REFERENCES}

(1) L. Muldawer: J. Appl. Phys., 37 (1966), 2062.

(2) M. E. Brooks and R. W. Smith: Scripta Met., 3 (1969), 667.
(3) Y. Murakami, N. Nakanishi and S. Kachi: Acta Met., 19 (1971), 93.

(4) Y. Murakami, S. Kachi, N. Nakanishi and H. Takehara: Acta Met., 19 (1971), 97.

(5) H. J. McQueen and G. C. Kuczynski: Trans. Met. Soc. AIME, 215 (1959), 619.

(6) R. G. Davies: Trans. Met. Soc. AIME, 230 (1964), 903.

(7) R. Feder and R. W. Cahn: Phil. Mag., 5 (1960), 343.

(8) K. Oki, M. Hasaka and T. Eguchi: Trans. JIM, 14 (1973), 8.

(9) G. E. Poquette and D. E. Mikkola: Trans. Met. Soc. AIME, 245 (1969), 743.

(10) G. Gupta and D. S. Lieberman: Ordered Alloys, Proc. 3rd Borton Landing Conf., Claitor's Publ. Div. (1970), p. 195.

(11) Y. Murakami, Y. Watanabe and S. Kachi: Trans. JIM, 21 (1980), 714.

(12) H. Kubo and K. Shimizu: Trans. JIM, 17 (1976), 330.

(13) P. A. Beck, J. C. Kremer, L. J. Demer and M. L. Holzworth: Trans. AIME, 175 (1948), 372.

(14) M. S. Wechsler: Acta Met., 5 (1957), 150.

(15) N. Nakanishi, K. M. Thein and C. M. Wayman: J. Appl. Phys., 34 (1963), 2847.

(16) M. J. Koczak, H. Herman and A. C. Damask: Acta Met., 19 (1971), 303.

(17) J. S. Clark and N. Brown: Acta Met., 19 (1971), 291.

(18) S. G. Cupchallc and N. Brown: Acta Met., 19 (1971), 657. 